\title{
Second-order Phase Transition Behavior in a Polymer above the Glass Transition Temperature
}

\author{
Mitsuru Ishikawa, * Taihei Takahashi, Yu-ichiro Hayashi, Maya Akashi, Takayuki Uwada \\ Department of Chemistry, Faculty of Science, Josai University, 1-1 Keyaki-dai, Sakado, \\ Saitama 350-0295, Japan
}

\begin{abstract}
Glass transition was primarily considered to be not phase transition; however, it has similarity to the second-order phase transition. Recent single-molecule spectroscopy developments have prompted re-investigating glass transition at the microscopic scale, revealing that glass transition includes phenomena similar to secondorder phase transition. They are characterized by microscopic collective polymer motion and discontinuous changes in temperature dependent relaxation times, later of which is similar to critical slowing down, within a temperature window that includes the polymer calorimetric glass transition temperature. Considering that collective motion and critical slowing down are accompaniments to critical phenomena, second-order phase transition behavior was identified in polymer glass transition.
\end{abstract}

\section{INTRODUCTION}

Single-molecule spectroscopy (SMS) based on fluorescence ${ }^{1}$ has opened a new era for exploring the molecular basis for cell biology, ${ }^{1}$ analytical chemistry, ${ }^{2}$ biochemistry and biophysics,,${ }^{1,3}$ organic synthesis and drug discovery, ${ }^{4}$ surface specific chemical reactions, ${ }^{5}$ and polymer dynamics. ${ }^{6-10}$ Now SMS has matured to where it offers a versatile tool to help advance many sciences and technologies. This progress has brought expectation that unexplored polymer dynamics may be found by SMS above the calorimetric glass transition temperature $T_{\mathrm{g}}$ and below the melting point $T_{\mathrm{m}}$, typically $T_{\mathrm{g}}+10-20 \mathrm{~K}$. This state above $T_{\mathrm{g}}$ is generally known as metastable or super-cooled liquids, ${ }^{11}$ regardless of polymers or monomers, in contrast to stable liquids above $T_{\mathrm{m}}$. Molecular level understanding regarding the extraordinarily increased relaxation times $\tau_{\mathrm{R}}$ by ten or more orders of magnitude at $T_{\mathrm{g}}$ has long been among a major unsolved problem in physics and chemistry. ${ }^{12,13}$ Our early challenge by SMS and time-resolved fluorescence spectroscopy using viscosity-sensitive fluorescence probes ${ }^{6,7,14,15}$ was to deepen glass transition understanding based on this fundamental query about extraordinarily increased $\tau_{\mathrm{R}}$ and related phenomena.

The present research was planned to re-examine the earlier achievements and to search something novel beyond previous knowledge with improved temporal resolution (from $10 \mathrm{~s}$ to 36 $\mathrm{ms})$. Before specifically considering present article challenge, we review temperature-dependent entropy $S$, heat capacity $C_{\mathrm{p}}$, and glass forming material viscosity $\eta$, to show how the challenge is related to phase transition like phenomena in the glass transition. Glass transition differs phase transition but apparently resembles second-order phase transition in the following three facts. This statement is just we mean second-order phase transition behavior in the present article.

First, glass transition is not first-order phase transition but exhibits second-order phase transition behavior in the sense that liquid $S$ profile joins the glass profile at $T_{\mathrm{g}}$. Figure 1A shows that temperature dependent $S$ continues from liquid to glass state without discontinuous transition at $T_{\mathrm{g}}$, although the slopes differ either side of $T_{\mathrm{g}}$.

Second, discontinuous change, rather than abrupt transition, in temperature-dependent $C_{\mathrm{p}}$ shows that glass transition apparently resembles second-order phase transition. Temperature dependent $C_{\mathrm{p}}=T(\partial S / \partial T)_{\mathrm{p}}$ has been evaluated by differential scanning calorimetry (DSC) to 
identify $T_{\mathrm{g}}$, where a single staircase step in $\Delta C_{\mathrm{p}}$ occurs, due to the discontinuous change in $(\partial S / \partial T)_{\mathrm{p}}$ between liquid and glass state. The transition interval $\Delta T$ associated with $\Delta C_{\mathrm{p}}$ step in glass transition is rather small, typically $10 \mathrm{~K}$ for many glass forming materials. ${ }^{16}$ Generally, $\Delta C_{\mathrm{p}}$ step characterizes the second-order phase transition. ${ }^{17}$

Last, the Vogel-Fulcher-Tammann (VFT) equation (eq 1) suggests that $\eta$ diverges at $T_{\mathrm{o}}$ near critical temperature $T_{\mathrm{c}}$ in the second-order phase transition. Figure 1B shows an Arrhenius profile for $\eta$ temperature dependence for glass forming materials sometimes called Angell plot. ${ }^{18}$ Nonlinear, or non-Arrhenius Angell plot curves fit the VFT relationship for $\eta$ between $T_{\mathrm{g}}$ and $T_{\mathrm{g}}+50$ $\mathrm{K}^{16}$ by

$$
\log \left(\eta / \eta_{\infty}\right)=B /\left(T-T_{o}\right),
$$

where $\eta_{\infty}$ is the limiting viscosity at infinite temperature, $T_{\mathrm{o}}$ is the Vogel temperature (several tens $K$ below $\left.T_{\mathrm{g}}\right)^{16}$, and $B$ and $T_{\mathrm{o}}$ are empirical parameters fitted from experimental observations.

We learn further glass transition fundamentals to understand present article backgrounds. Figure 1A includes liquid and crystal separation below $T_{\mathrm{m}}$, glass formation at $T_{\mathrm{g}}$, and cooling-rate dependent $T_{\mathrm{g}}$ and $T_{\mathrm{g}}^{\prime}$; the difference between them can be $3-5 \mathrm{~K}$ on changing cooling rate by an order of magnitude. ${ }^{11}$ Although cooling-rate dependence for $T_{\mathrm{g}}$ repels glass transition being a phase transition, glass transition exhibits second-order phase transition behavior, as exemplified above three representative instances.
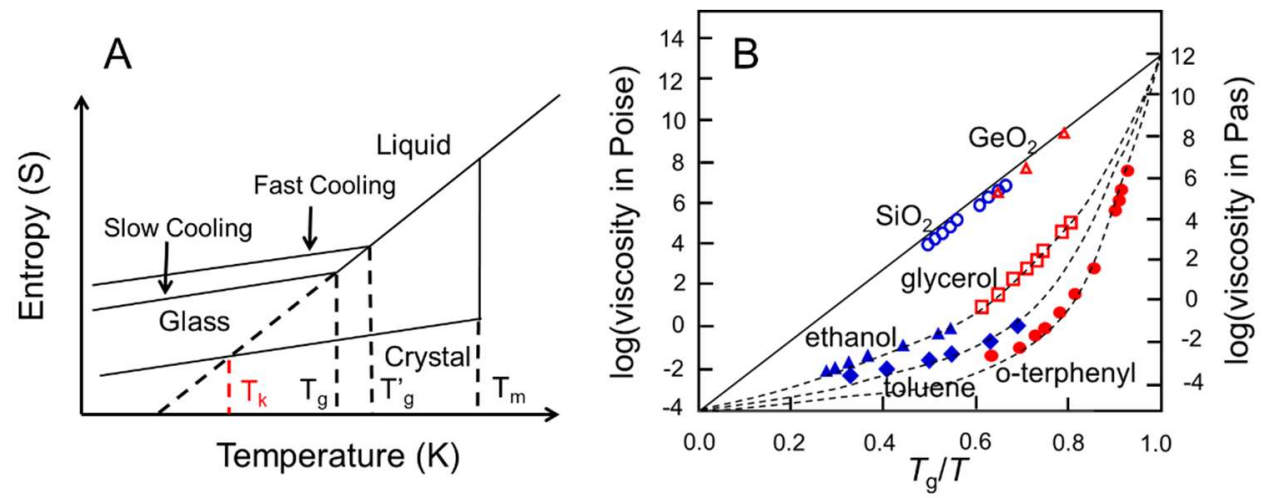

Figure 1. Glass transition fundamentals. (A) Temperature dependent entropy for glass forming materials including monomers and polymers, showing four important temperatures: melting point $T_{\mathrm{m}}$, cooling-rate dependent glass transition temperatures $T_{\mathrm{g}}$ and $T_{\mathrm{g}}$, and Kauzmann temperature $T_{\mathrm{k}}$; (B) Simplified Angel plot. (A) was adapted from an article (https://www.jps.or.jp/books/gakkaishi/2016/05/71-0570fushigi 09.pdf.) by the Physical Society of Japan. (B) was adapted from ref 18.

Figure 1A suggests another second-order phase transition behavior, showing that we can expect temperature to be crossed by the crystal and extrapolated liquid lines called the Kauzmann temperature $T_{\mathrm{k}}$. Below $T_{\mathrm{g}}$, the extrapolated liquid line falls beneath the crystal line, violating a thermodynamic fundamental that crystal entropy should be lower than that of liquid. Surprisingly, $T_{\mathrm{o}}$ is close to $T_{\mathrm{k}}$ for many glass forming materials, typically $0.9<T_{\mathrm{k}} / T_{\mathrm{o}}<1.1{ }^{11}$ Hence, $T_{\mathrm{k}}$ is expected to be a real rather than imaginary temperature with substantial physical validity. The physics behind $T_{\mathrm{o}} \approx T_{\mathrm{k}}$ is challenging to address theoretically ${ }^{19}$ and experimentally, ${ }^{13}$ and hence to understand glass transition essentials, but it is outside the present article scope. Temperature dependent $S, C_{\mathrm{p}}$, and $\eta$ for glass forming materials thus offers possibilities to observe secondorder phase transition behavior above $T_{\mathrm{k}}$, or near $T_{\mathrm{g}}$.

Figure 1B tells us a glass transition criterion that $\eta$ for many glass forming materials presents $\approx 10^{12}$ Pas at $T=T_{\mathrm{g}}$. Additionally, $\tau_{\mathrm{R}}$ is approximately proportional to $\eta$, that is $\eta \approx G_{\mathrm{g}} \cdot \tau_{\mathrm{R}}$, where $G_{\mathrm{g}}$ is the glass modulus, $10^{9}-10^{12} \mathrm{~Pa} .{ }^{16} \mathrm{In}$ practice, $\tau_{\mathrm{R}} \approx 100 \mathrm{~s}$ is accepted for glass forming materials at $T_{\mathrm{g}}{ }^{11,20}$ as $G_{\mathrm{g}}=10^{10} \mathrm{~Pa}$ as a common rule of thumb for glassware workers. These 
values for $\eta$ and $\tau_{R}$ are known as glass transition dynamics empirical criteria.

We further examine glass transition and second-order phase transition related concepts to provide more direct background for the present article, such as glass forming material $\tau_{\mathrm{R}}$ in $\alpha$ process rather than $\eta$ in dielectric loss spectroscopy, collective molecular motion and cooperatively rearranging region (CRR) near $T_{\mathrm{g}}$, and critical phenomena associated with secondorder phase transition.

Dielectric loss spectroscopy has evaluated temperature dependent $\tau_{\mathrm{R}}$ or relaxation frequencies $\omega$, rather than $\eta$ measurement, and characterized glass transition through the extraordinary $\omega \approx$ $1 / \eta$ slowdown near $T_{\mathrm{g}}$. In this spectroscopy, a single peak occurs at $\omega$ for liquid of glass-forming material above $T_{\mathrm{m}}$, that is, one relaxation mechanism dominates. Cooling the liquid below the crossover temperature $T_{\text {cross }} \approx 1.2 T_{\mathrm{g}}$, expected from mode-coupling theory (MCT), ${ }^{11,}{ }^{20}$ splits a single peak into faster and slower peaks. ${ }^{11}$ Dynamics characterized by the slower frequency is called $\alpha$ process and that characterized by the faster frequency is called $\beta$ process. The $\alpha$ process exhibits non-Arrhenius behavior and disappears at $T_{\mathrm{g}}$, whereas $\beta$ process continues Arrhenius behavior below $T_{\mathrm{g}}$. The $\tau_{\mathrm{R}}$ for $\alpha$ process, typically $0.1-1.0 \mathrm{~s}$, at $T_{\mathrm{g}}+10-20 \mathrm{~K}$, is much slower ( $>$ $10^{7}$-fold) than for $\beta$ process at $T_{\mathrm{g} .}{ }^{11,16}$ Because our SMS technique lacked temporal resolution to detect $\beta$ process, we only considered $\alpha$ process assigned to polymer main chain segmental Brownian motion ${ }^{21}$ and characterized by cooperative nature.

Collective or cooperative molecular motion results from the non-Arrhenius Angell plot. Surrounding molecules form an energy barrier that a molecule of interest must overcome to move away. This barrier increases with reducing temperature, that is, the surrounding molecules behave collectively to affect molecule movement. Thus, non-Arrhenius behavior for $\eta$ or $\omega$ leads to cooperative, or collective motion for glass forming materials as $\alpha$ process, and ultimately contributes to extraordinary $\tau_{\mathrm{R}}$ slowdown. In addition, distinct spatial domains develop along with $\alpha$ process slowdown, generating spatial heterogeneity in glass forming materials. Heterogeneity size was estimated at $1-5 \mathrm{~nm}$ near $T_{\mathrm{g}}$ for many glass forming materials. ${ }^{16,22-24}$ It is the size of $\mathrm{CRR}$, or characteristic length $\xi_{\alpha}$, that is, average CRR diameter.

Fluid critical phenomena near $T_{\mathrm{c}}$ are characterized by highly fluctuating density in collective molecular motion, correlation length $\xi_{\alpha}$ divergence, and critical slowing down. These phenomena are all correlated with each other and well exemplified by the $\mathrm{CO}_{2}$ critical opalescence, ${ }^{17}$ which generates a change in transparency to turbid appearance near $T_{\mathrm{c}}$. Thus, $\mathrm{CO}_{2}$ molecules behave collectively in the size beyond or close to visible light wavelengths $400-700 \mathrm{~nm}$, and thus collective $\mathrm{CO}_{2}$ molecules present vigorous fluctuations detectable by light scattering measurement, much slower than $\mathrm{CO}_{2}$ molecular motions.

Based on the glass transition and second-order phase transition related fundamentals above noted, we outline two major findings in the present article, demonstrating polymer second-order phase transition behavior near $T_{\mathrm{g}}$, much higher than $T_{\mathrm{k}}$. They were observed in a temperature window including $T_{\mathrm{g}}$ but below $T_{\text {cross. }}$. One is substantial evidence for poly(vinyl acetate) PVAC collective motion. The other is the discontinuous change in temperature dependent PVAC average relaxation time $\left\langle\tau_{\mathrm{R}}>\right.$, which looks critical slowing down in critical phenomena associated with second-order phase transition considering simultaneous collective motion occurrences. The first one was characterized by distinct cosine waveforms for autocorrelation functions $C(\tau)$ evaluated from single molecule Cy3 fluorescence trajectory $I_{\mathrm{f}}(\mathrm{t})$. These findings appeared beyond the known theoretical framework for glass transition and related phenomena, such as MCT. The two major findings provided evidence suggesting that glass transition includes second-order phase transition behavior above $T_{\mathrm{g}}$, although glass-transition is not a phase transition. However, correlation length $\xi_{\alpha}$ divergence as PVAC critical opalescence was not found in the present work.

We selected PVAC due to its $T_{\mathrm{g}}$ above room temperature $\left(20-25^{\circ} \mathrm{C}\right)$ and below $40{ }^{\circ} \mathrm{C}$ suitable for the present temperature controlling setup (see Instruments used for SMS in Materials and Methods in Supporting Information, SI). Viscosity-sensitive Cy3 was used in SMS, ${ }^{7}$ because its 
fluorescence quantum efficiency $\Phi_{\mathrm{f}}$ increased with increasing solvent viscosity: $\Phi_{\mathrm{f}}=0.042$ in fluid $(297 \mathrm{~K})$ and 0.94 in rigid $(77 \mathrm{~K})$ ethanol solution, respectively. ${ }^{25}$
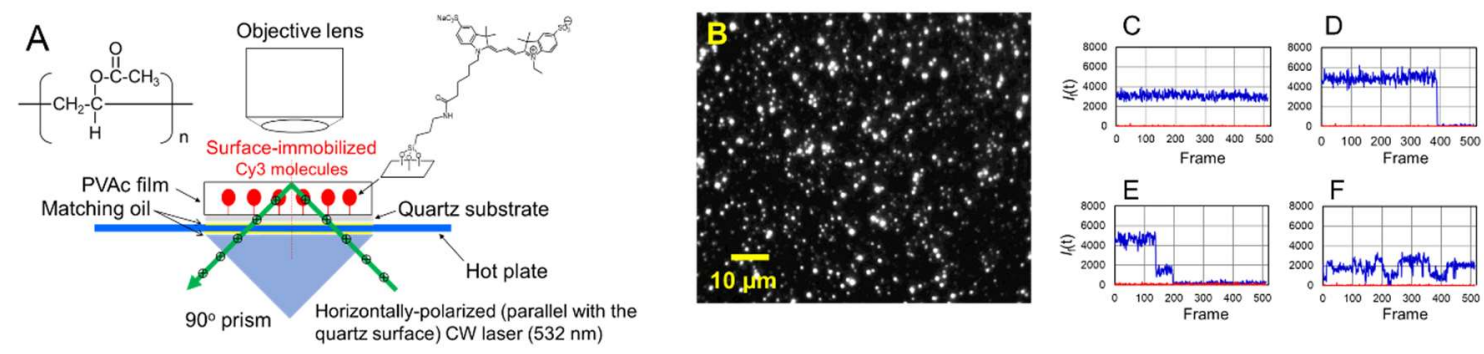

Figure 2. Single-molecule spectroscopy (SMS) fundamentals. (A) Experimental setup for SMS used in the present work: single Cy3 molecules covalently immobilized on a quartz surface with PVAC overlay, light microscope (shown as objective lens), quartz prism for horizontally polarized (parallel with the sample surface) evanescent illumination, and glass hot plate transparent in the visible region; (B) wide-field fluorescence image averaged over 512 frames $\left(36 \mathrm{~ms} /\right.$ frame) followed by background subtraction. Fluorescence trajectory $I_{\mathrm{f}}(t)$ for single Cy3 molecules at room temperature $\left(24.5^{\circ} \mathrm{C}\right)$ showing $(\mathbf{C})$ no photobleaching, $(\mathbf{D})$ one-step photobleaching, $(\mathbf{E})$ two-step photobleaching, and (F) substantial fluctuation.

\section{RESULTS}

Figure 2A shows the SMS setup we employed in the present work, with which we obtained SMS fundamental (Figures 2B-2F) and key observations that support the first major finding, PVAC collective motion. After $\left\langle\tau_{R}>\right.$ evaluation we found the second major finding, discontinuous $<\tau_{R}>$ change in a specific temperature window in which PVAC collective motion was activated.

We employed horizontally polarized excitation (Figure 2A) in the present SMS experiments due to $\mathrm{Cy} 3$ absorption transition moment being highly oriented on a substrate surface at room temperature $\left(23.8^{\circ} \mathrm{C}\right.$ ) (Figures S1A and S1B) and an elevated temperature $\left(56.3{ }^{\circ} \mathrm{C}\right.$ ) (Figures S1C and S1D). To dispel concerns about SMS application to glass transition research using surface-immobilized single molecule, we examined SMS fluorescence intensities and $C(\tau)$ waveforms with and without PVAC overlay. Enhanced fluorescence intensities (2.16-fold, Figures S2A and S2B) with PVAC overlay and no cosine $C(\tau)$ without PVAC overlay (Figures S3A-S3C) support single $\mathrm{Cy} 3$ capabilities to detect changes in PVAC environment accompanied by glass transition.

We expect those who have concerns about the polarized excitation and the use of surfaceimmobilized probe molecules to consult related descriptions in Supplementary Text in SI.

\section{Single Cy3 molecule observation, fundamentals}

Figure 2B shows a single molecule Cy3 fluorescence image with PVAC overlay at $24.5^{\circ} \mathrm{C}$. This image was processed by averaging 512 frames ( $36 \mathrm{~ms} /$ frame), followed by background subtraction. One hundred fluorescent spots were selected to evaluate photo-bleached spots in the 512-frame acquisition: $17-23 \%$ spots were photo-bleached with PVAC overlay, whereas 35-40\% spots were photo-bleached without PVAC overlay at $22-25{ }^{\circ} \mathrm{C}$. More than $70 \%$ spots were temporally stable with PVAC overlay (Figure 2C).

Single staircase photobleaching (Figure 2D) confirmed that the phenomenon was due to single Cy3 molecules, with rare $(0.1 \%$ maximum) two-step photobleaching occurrences (Figure 2E). This means that one brighter fluorescent spot included two Cy3 molecules. A few $(\approx 5 \%)$ highly fluctuating spots also present (Figure 2F). Ideally, we should follow the identical single Cy3 molecules from start to finish in all SMS measurement; however, photobleaching prohibited the ideal measurement. This is a fundamental SMS limitation.

Fluorescence intensity varied appreciably from spot to spot (Figure 2B). This was partly due to 
photo-bleached spots in the image acquisition and excitation light polarization, but mainly due to highly heterogeneous polymer structures.

\section{Temperature dependent single molecule fluorescence trajectories}

We observed $I_{\mathrm{f}}(t)$ temporal fluctuations for 20-30 fluorescent spots that survived from photobleaching in 512-frame video acquisition in heating and cooling experiment. In a pair of heating and cooling experiments 20 to 30 fluorescent spots were evaluated at each temperature. We have two or more pairs heating and cooling experiments. Such repeated experiments exist behind our view about the experimental findings presented in this article.

Temperature dependent $C(\tau)$ (Figures 3A-3F for heating and Figures S4A-S4F for cooling) evaluated from $I_{\mathrm{f}}(t)$ included remarkable $C(\tau)$ cosine waveforms, hence providing evidence for collective PVAC motion above $T_{\mathrm{g}}$. This is the first major finding in the present article. Collective motion was deduced from the generalized Langevin equation (GLE ${ }^{26}$ in Derivation of the $C(\tau)$ cosine waveform from generalized Langevin equation in Discussion and Supplementary Text in SI.

Figures $3 \mathrm{~A}-3 \mathrm{E}$ illustrate representative $I_{\mathrm{f}}(t), C(\tau)$, and power spectrum $J(v)$, Fourier transform of $C(\tau)$, while heating $24.5-64.9{ }^{\circ} \mathrm{C}$. Although temperatures were elevated in a step-by-step manner: $24.5,28.3,29.6,32.0,35.6,37.8,41.9,45.4,51.9,56.4,60.2$, and $64.9^{\circ} \mathrm{C}$ at $0.2 \mathrm{~K} / \mathrm{min}$, these figures include limited temperatures to highlight the remarkable $C(\tau)$ cosine waveform at 41.9 and $56.4{ }^{\circ} \mathrm{C}$ and temperature dependent specific sub-second frequencies simultaneously occurred with the enhanced and reduced $J(v)$ amplitude: $0.109 \mathrm{~Hz}(0.096), 0.217 \mathrm{~Hz}(0.472)$, and $0.542 \mathrm{~Hz}(0.200)$, where parentheses include the highest $J(v)$ value obtained at 29.6, 41.9, and $56.4{ }^{\circ} \mathrm{C}$, respectively.

Those being interested in the intermittent $I_{\mathrm{f}}(t)$ temporal fluctuation mechanism should consult related descriptions in Supplementary Text in SI.

Cosine waveform $C(\tau)$, the enhanced and reduced $J(v)$ amplitudes, and the increasing frequencies in $J(v)$ with increasing temperature prompted us to select and classify $C(\tau)$. Figure $4 \mathrm{~A}$ and Table S1A summarize occurrences for each $C(\tau)$ type denoted in Figures 3A-3E and S4A$\mathrm{S} 4 \mathrm{E}$ at the above temperatures. Classification criteria for $I_{\mathrm{f}}(t)$ are as follows: Type I for stable $I_{\mathrm{f}}(t)$ and no specific peaks in $J(v)$, Type II for fluctuating $I_{\mathrm{f}}(t)$ but no touching the base line with a small $(<0.10)$ single peak in $J(v)$, Type III for fluctuating $I_{\mathrm{f}}(t)$ touching base line with a prominent $(0.20<)$ single peak in $J(v)$, and Type IV for fluctuating $I_{\mathrm{f}}(t)$ touching the base line with a prominent $(<0.20)$ single or multiple peaks in $J(v)$, and Type $\mathrm{V}$ for fluctuating $I_{\mathrm{f}}(t)$ touching the base line without prominent peaks $(0.10<)$ in $J(v)$. Waveforms $C(\tau)$ and $J(v)$ in Type $\mathrm{V}$ are similar to those of Type I. For example, $C(\tau) \mathrm{s}$ at $56.4{ }^{\circ} \mathrm{C}$ include Type II, III, IV, and V occurrences (Figure 4A and Table S1A). The wide diversity is acceptable considering the intrinsically inhomogeneous polymer structures probed by SMS.

Because $J(v)$ amplitudes can be a measure of purity in cosine waveforms, we compared the highest $J(v) 0.472$ at $41.9{ }^{\circ} \mathrm{C}$ with amplitude $0.930(0.977 \mathrm{~Hz})$ computed by Fourier transform of the noise-free and damping-free unitary cosine wave at $1 \mathrm{~Hz}(\cos 2 \pi \mathrm{t})$ with $36 \mathrm{~ms}$ bin time, showing $>50 \%(0.472 / 0.930)$ purity for $C(\tau)$ in Figure 3C.

To avoid possible misunderstandings that all observed $C(\tau)$ continued forever, Figure 3F shows $C(\tau)$ damped oscillation reproduced by the sum of cosine functions multiplied by a single exponential decay amplitude, which was classified into Type IV. Damped $C(\tau)$ oscillation was usually observed in Type III and IV $C(\tau)$.

Figures 3A-3E show representative $J(v)$, with highest amplitudes from 0.096 at $29.6{ }^{\circ} \mathrm{C}$ to 0.200 at $56.4{ }^{\circ} \mathrm{C}$ and maximum overall amplitude 0.472 at $41.9^{\circ} \mathrm{C}$. To ensure this tendency was fundamental, we evaluated $C_{\mathrm{av}}(\tau)$ by averaging twenty $C(\tau)$ waveforms survived from photobleaching and computed $J_{a v}(v)$ from $C_{\text {av }}(\tau)$ in heating experiment (Figures 5A-5L). Maximum $J_{\mathrm{av}}(v)$ at each temperature has $J_{\mathrm{av}}{ }^{\max }$ peak at $41.9^{\circ} \mathrm{C}$. Figures $3 \mathrm{C}$ and $3 \mathrm{~F}$ include $C(\tau)$ 
damped oscillations fitted with fitting functions $C(\tau)=C_{0} \exp \left(-t / C_{1}\right)\left\{C_{2} \cos \left(C_{3} t+C_{4}\right)\right\}+C_{5}$ and $C(\tau)=C_{0} \exp \left(-t / C_{1}\right)\left\{C_{2} \cos \left(C_{3} t+C_{4}\right)+C_{5} \cos \left(C_{6} t+C_{7}\right)+C_{8} \cos \left(C_{9} t+C_{10}\right)+C_{11} \cos \left(C_{12} t+C_{13}\right)\right\}$ $+C_{14}$, respectively.
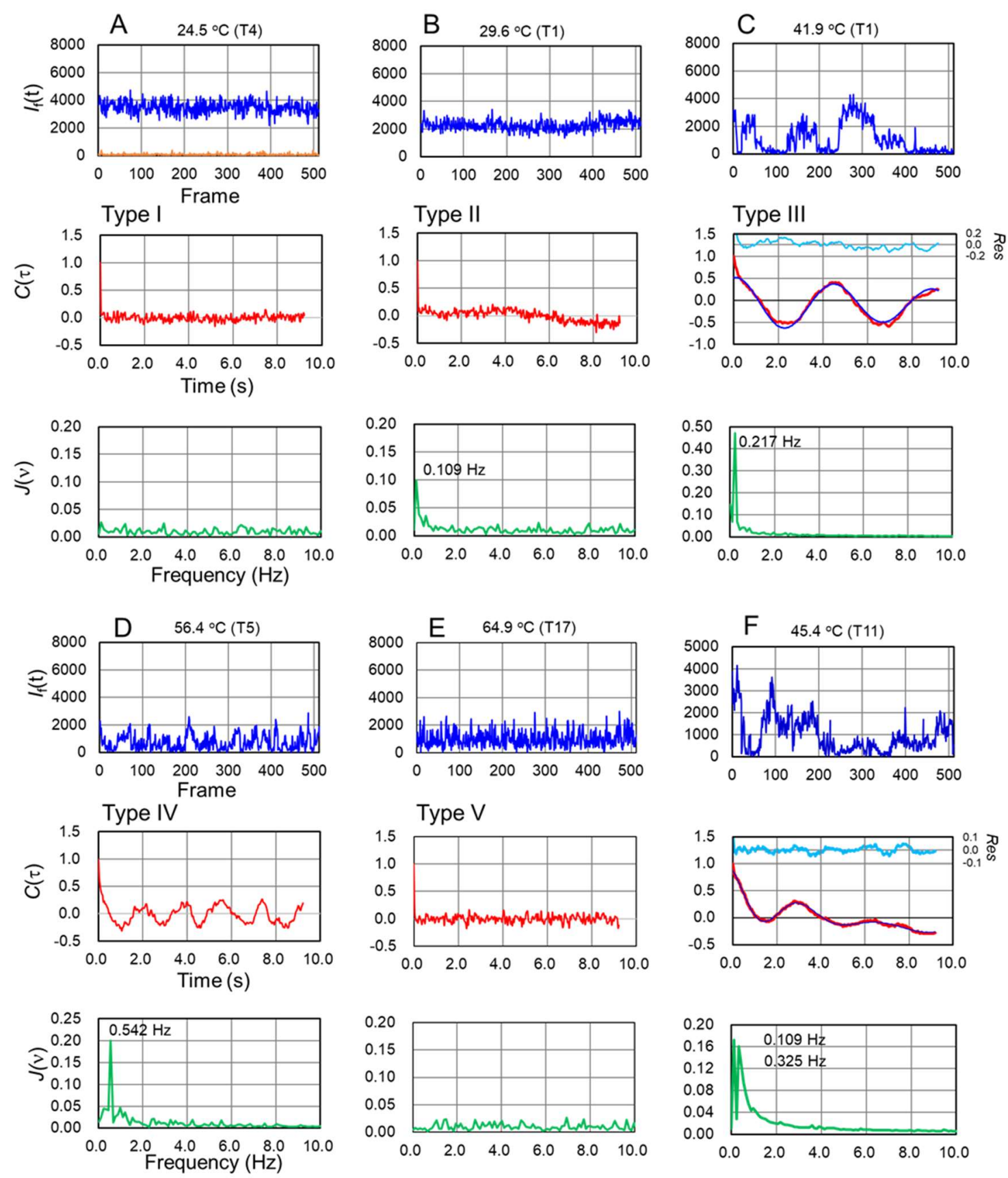

Figure 3. Temperature dependent representative fluorescence trajectories, $I_{\mathrm{f}}(t)$; autocorrelation functions, $\boldsymbol{C}(\boldsymbol{\tau})$; and power spectra, $\boldsymbol{J}(\boldsymbol{v})$. They were observed from single Cy3 molecules with PVAC overlay from heating experiment $(0.2 \mathrm{~K} / \mathrm{min})$ at (A) 24.5, (B) 29.6, (C) 41.9, (D) 56.4, (E) 64.9, and (F) $45.4^{\circ} \mathrm{C}$. Sets (C) and (F) include the residue (light blue curve) between experimental (red curve) and computed (blue curve) traces. Labels Type I to Type $\mathrm{V}$ are for $C(\tau)$ classification. Video images generating these data were captured with horizontally polarized excitation and averaged over 512 frames $(36 \mathrm{~ms} /$ frame $)$ followed by background subtraction. 
In Figure 3F, we used the fitting function including four cosine functions against immediate expectation from $J(v)$ : two cosine functions including two distinct frequencies 0.109 and 0.325 $\mathrm{Hz}$ in $J(v)$. This is likely due to tailing in the $0.325 \mathrm{~Hz}$ spectrum, including unresolved spectra contribution in $J(v)$. In a similar way to Figures $3 \mathrm{~A}-3 \mathrm{~F}$, we present Figures $\mathrm{S} 4 \mathrm{~A}-\mathrm{S} 4 \mathrm{~F}$ to show $I_{\mathrm{f}}(t), C(\tau)$, and $J(v)$ cooling from 64.6 to $25.3{ }^{\circ} \mathrm{C}$ at $0.2-0.3 \mathrm{~K} / \mathrm{min}$. Figure $4 \mathrm{~B}$ and Table S1B summarize Type I to $\mathrm{V}$ occurrences at each temperature for cooling.

Symbols in Figures 3A-3F, S3A-S3C, and S4A-S4F, for example T1 and T17, identify fluorescence spots in the video images mainly for authors' convenience to easily trace highlighted location on the original video images.
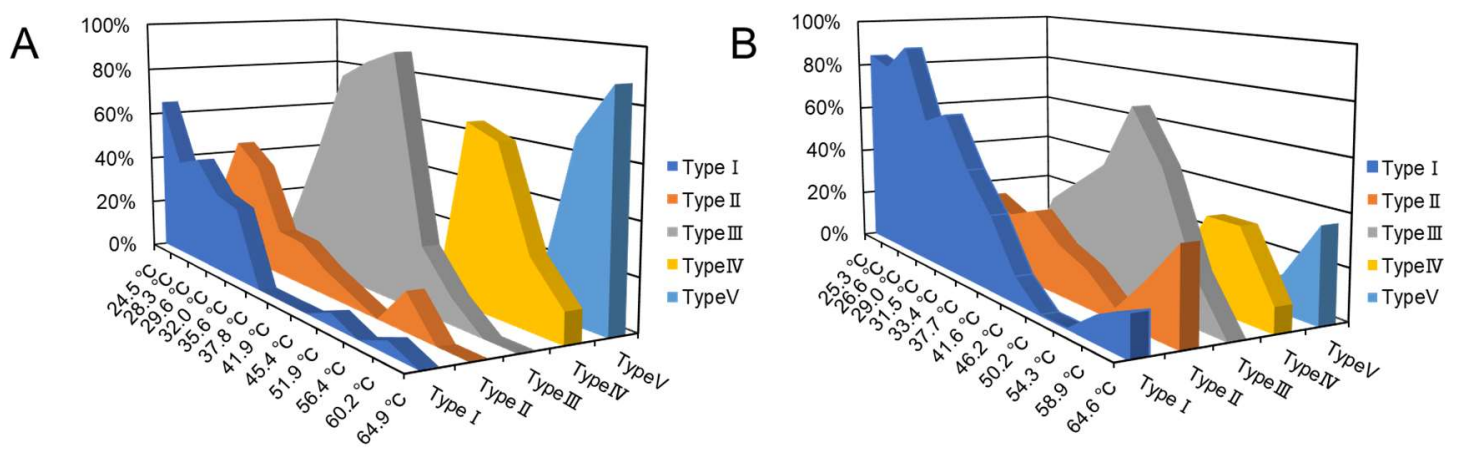

Figure 4. Autocorrelation $\boldsymbol{C}(\tau)$ classification into six types for each observed temperature. Type I to Type $\mathrm{V}$ occurrences for $(\mathbf{A})$ heating $24.5-64.9^{\circ} \mathrm{C}(0.2 \mathrm{~K} / \mathrm{min})$ and $(\mathbf{B})$ cooling from $64.6-25.3{ }^{\circ} \mathrm{C}(0.2-0.3 \mathrm{~K} / \mathrm{min})$ are summarized. Twenty fluorescent spots from single Cy3 molecules with PVAC overlay were collected at each temperature. Video images generating these data were captured with horizontally polarized excitation and averaged over 512 frames ( $36 \mathrm{~ms} /$ frame) followed by background subtraction.

\section{Relaxation time evaluation from autocorrelation functions}

Representative $C(\tau)$ showing a cosine waveform and $J(v)$ are presented in Figures 3C and 3D for heating and in Figures S4B and S4C for cooling. However, $J(v)$ was insufficient to evaluate damped $C(\tau)$ oscillations, such as those in Figures 3F and S4F; $J(v)$ extracted only oscillatory characteristics from $C(\tau)$. Thus, $\tau_{\mathrm{R}}$ is required to extract damping behavior from $C(\tau)$. For this end, we used $C_{\mathrm{av}}(\tau)$ to cancel oscillatory characteristics, in particular frequencies higher that $0.1 \mathrm{~Hz}$, from $C(\tau)$. The use of $C_{\text {av }}(\tau)$ made it possible to use the Kohlrausch-Williams-Watts (KWW) function $\exp \left[-\left(t / \tau_{\mathrm{R}}\right)^{\beta}\right]^{11,16}(0<\beta \leq 1)$, which has widely been used for $\alpha$ process relaxation analysis.

We computed $\tau_{\mathrm{R}}$ from $C_{\mathrm{av}}(\tau)$ using

$$
C_{a v}(\tau)=C_{o} \exp \left[-\left(t / C_{1}\right)^{C_{2}}\right]+C_{3} \cos \left(C_{4} t+C_{5}\right)+C_{6}
$$

where $C_{0}, C_{1}=\tau_{\mathrm{R}}, C_{2}=\beta, C_{3}, C_{4}, C_{5}$, and $C_{6}$ are fitting parameters. Note that the unity at $\tau=0$ was removed from $C_{\mathrm{av}}(\tau)$ to allow $\tau_{\mathrm{R}}$ computation easy; instead, the value at $\tau=1$ was pasted as the value at $\tau=0$. The KWW function extracts $\tau_{\mathrm{R}}$ distribution in $C_{\mathrm{av}}(\tau)$ through the parameter $\beta$. The evaluated $C_{1}=\tau_{\mathrm{R}}$ is then converted into $<\tau_{\mathrm{R}}>$ using

$$
\left\langle\tau_{R}\right\rangle=\int_{0}^{\infty} e^{-\left(t / \tau_{R}\right)^{\beta}} d t=\left(\tau_{R} / \beta\right) \Gamma(1 / \beta)
$$

where $\Gamma$ is the gamma function, and the area under the KWW function in eq 3 denotes $<\tau_{R}>$. 9 Figures $5 \mathrm{~A}-5 \mathrm{~L}$ show all the temperature dependent $C_{\mathrm{av}}(\tau),<\tau_{\mathrm{R}}>$, and $J_{\mathrm{av}}(v)$, Fourier transform of $C_{\text {av }}(\tau)$. The computed $\beta$ ranged 0.45 to 1.0 and 0.36 to 1.0 for heating in Figures $5 \mathrm{~A}-5 \mathrm{~L}$ and for cooling in Figure S5A-S5L at 64.6, 58.9, 54.3, 50.2, 46.2, 41.6, 37.7, 33.4, 31.5, 29.0, 26.6, and 
$25.3{ }^{\circ} \mathrm{C}$ at $0.2-0.3 \mathrm{~K} / \mathrm{min}$. In the subsequent section, we employ $J_{\mathrm{av}}(v)$ to find specific temperature windows showing highest $J_{\mathrm{av}}(v)$ intensities in heating and cooling experiments.
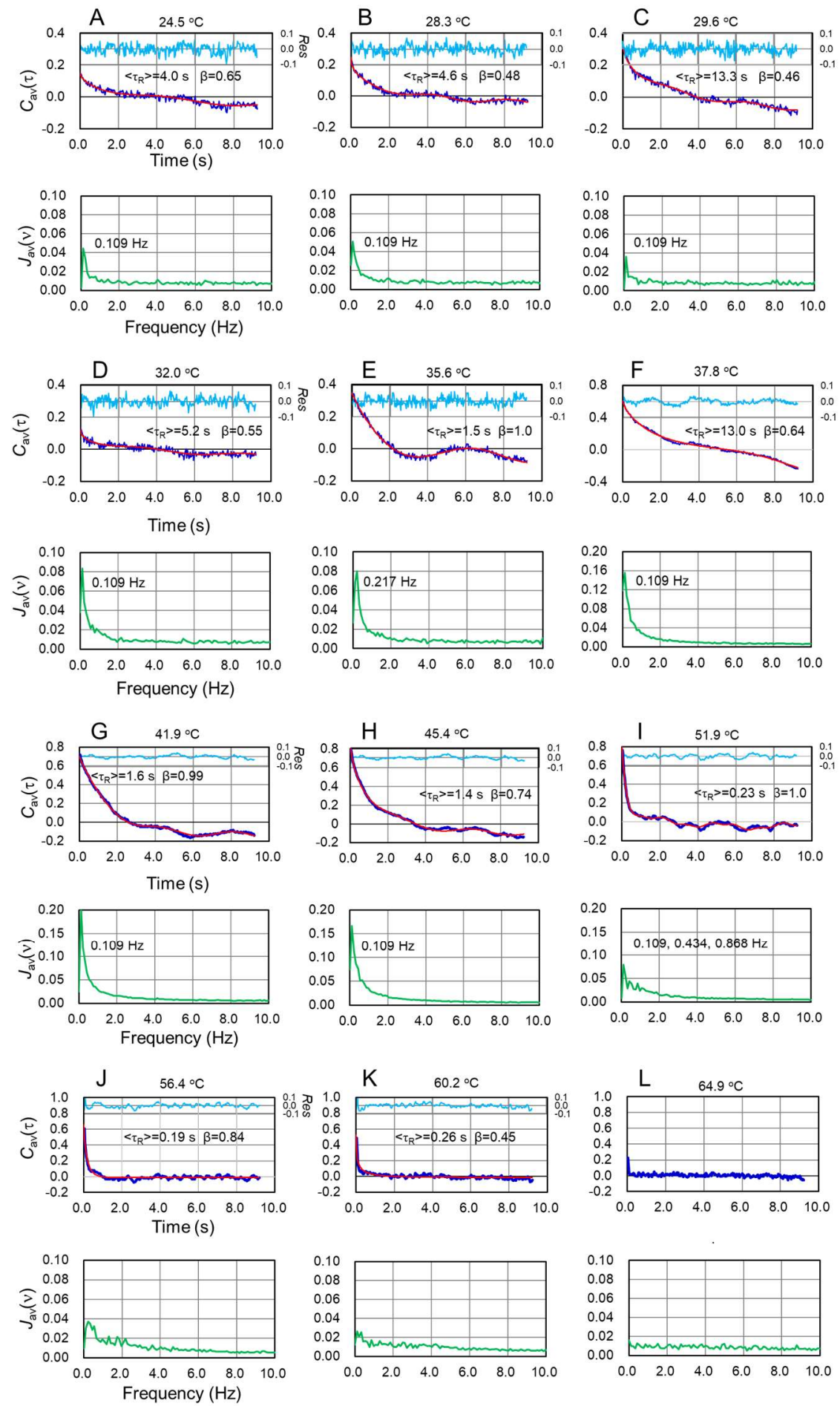

Figure 5. Average autocorrelation functions, $\boldsymbol{C}_{\mathrm{av}}(\tau)$ and power spectra, $\boldsymbol{J}_{\mathrm{av}}(v)$. They were observed for heating $(0.2 \mathrm{~K} / \mathrm{min})$ from single Cy3 molecules with PVAC overlay and computed from twenty single Cy3 molecules free from photobleaching at (A) 24.5, (B) 28.3, (C) 29.6, (D) 32.0, (E) 35.6, (F) 37.8, (G) 41.9, (H) 45.4, (I) 51.9, (J) 56.4, (K) 60.2, and (L) $64.9^{\circ} \mathrm{C}$. In (L), $<\tau_{\mathrm{R}}>$ evaluation was halted due to inability to identify decay in $C_{\text {av }}(\tau)$. Each $C_{\text {av }}(\tau)$ curve except for $(\mathbf{L})$ was fitted with eq 2 and includes calculated curve (red) and 
In Figures 3F and S4F, we used the product of one single exponential function and one cosine function or cosine function summation to treat $I_{\mathrm{f}}(t)$ as a single damped oscillator in a conventional manner. In Figures 5A-5L and S5A-S5L, however, the sum of the KWW and a cosine function (eq 2) was used because the product did not generate satisfactory fitting between $C_{\mathrm{av}}(\tau)$ and the calculated one. Based on simple probability estimation, the unsatisfactory fitting using twofunction product is assumed to be the fact that "A and B" like the two-function product restricts the number of cases rather than "A or B" like two-function sum, where A and B represent the number of cases. Two-function product likely narrows satisfactory fitting possibilities.

\section{Temperature dependent relaxation times in a specific temperature window near $\boldsymbol{T}_{\mathrm{g}}$}

In this section, we first compared PVAC DSC measurements, temperature dependent $J_{\mathrm{av}}{ }^{\max }$, which is $J_{\text {av }}(v)$ maxima, and $\left\langle\tau_{\mathrm{R}}>\right.$ (Figures $5 \mathrm{~A}-5 \mathrm{~L}$ and Figure $\mathrm{S} 5 \mathrm{~A}-\mathrm{S} 5 \mathrm{~L}$ ) to find a temperature window in which $C_{\mathrm{av}}(\tau)$ oscillations along with $J_{\mathrm{av}}{ }^{\max }$ and $<\tau_{\mathrm{R}}>$ peaks were prominent, later of which are the second major finding.

Figures 6A and 6B show PVAC DSC measurements for heating and cooling (both at $5 \mathrm{~K} / \mathrm{min}$ ), respectively. We found three specific temperatures: $T_{1}=33.0^{\circ} \mathrm{C}$ (heating), $T_{2}=43.2^{\circ} \mathrm{C}$ (cooling), and $T_{3}=30.4{ }^{\circ} \mathrm{C}$ (cooling). We found $T_{\mathrm{g}}=36.5^{\circ} \mathrm{C}(309.5 \mathrm{~K})$ from the midpoint of the gap between extrapolated glass and melt lines in a cooling experiment. We found a PVAC $T_{\mathrm{g}}$ report showing 297-315 K, ${ }^{27}$ although no MW was specified. Generally, the higher the MW, the higher the $T_{\mathrm{g}}$. From commercially available PVAC, we assume that MW ranges $90,000-600,000$ in the report. ${ }^{27}$ From this speculation and the reported values, $T_{\mathrm{g}}=309.5 \mathrm{~K}$ we observed from MW 100,000 PVAC is reasonable as PVAC $T_{\mathrm{g}}$.

We found another temperature zone in Figure 6A, under the baseline a broken blue line above $T_{1}=33.0^{\circ} \mathrm{C}$, due to enthalpy overshoot, ${ }^{13}$ an endothermic process intrinsic to heating DSC. These phenomena always occurred in our observations, showing not only single dips (Figure 6A) but also multiple dips in different appearance for more than twenty DSC records. In contrast, no enthalpy overshoots were reproducibly observed for cooling experiments in Figure 6B.

The two peaks at 41.9 and $46.2{ }^{\circ} \mathrm{C}$ in Figures $6 \mathrm{C}$ and $6 \mathrm{D}$ for heating and cooling at $0.2-0.3$ $\mathrm{K} / \mathrm{min}$, respectively, are separated by $\approx 4 \mathrm{~K}$. This is likely due to hysteresis between heating and cooling. However, another pair of heating and cooling $(1.0 \mathrm{~K} / \mathrm{min})$ experiments in Figures $6 \mathrm{G}$ and $6 \mathrm{H}$ exhibited $\approx 9 \mathrm{~K}$ hysteresis at lower temperature for cooling. The heating and cooling experiments confirmed that $J_{a v}(v)$ amplitude reached the top between $T_{\mathrm{g}}$ and $T_{\mathrm{g}}+10 \mathrm{~K}$, rather than the hysteresis from heating and cooling. Hysteresis behavior differed from experiment to experiment including different heating and cooling rates.

Figures $6 \mathrm{C}$ and $6 \mathrm{E}$ compare temperature dependent $J_{\mathrm{av}}{ }^{\max }$ and $<\tau_{\mathrm{R}}>$ for heating. Temperature at $J_{\mathrm{av}}{ }^{\text {max }}$ peak $\left(41.9^{\circ} \mathrm{C}\right)$ is higher than that of $<\tau_{\mathrm{R}}>$ peak $\left(37.8^{\circ} \mathrm{C}\right)$ above $T_{\mathrm{g}}$. Figures $6 \mathrm{D}$ and $6 \mathrm{~F}$ show $J_{\mathrm{av}}{ }^{\max }$ and $\left\langle\tau_{\mathrm{R}}>\right.$ for cooling experiment. Temperature at $J_{\mathrm{av}}{ }^{\max }$ peak $\left(46.2^{\circ} \mathrm{C}\right)$ is again higher than that of $\left\langle\tau_{\mathrm{R}}\right\rangle$ peak $\left(41.6^{\circ} \mathrm{C}\right)$ above $T_{\mathrm{g}}$. Similarly, Figures $6 \mathrm{G}$ and 6I compare temperature dependent $J_{\mathrm{av}}{ }^{\max }$ and $<\tau_{\mathrm{R}}>$ for heating experiment. Temperature at $J_{\mathrm{av}}{ }^{\max }$ peak $\left(46.4^{\circ} \mathrm{C}\right)$ is equal to that of $\left\langle\tau_{\mathrm{R}}>\right.$ peak $\left(46.4{ }^{\circ} \mathrm{C}\right)$ above $T_{\mathrm{g}}$. Figures $6 \mathrm{H}$ and $6 \mathrm{~J}$ show $J_{\mathrm{av}}{ }^{\max }$ and $\left\langle\tau_{\mathrm{R}}>\right.$ for cooling experiment. Temperature at $J_{\mathrm{av}}{ }^{\text {max }}$ peak $\left(37.4^{\circ} \mathrm{C}\right)$ higher than that of $\left\langle\tau_{\mathrm{R}}\right\rangle$ peak $\left(32.8^{\circ} \mathrm{C}\right)$ below $T_{\mathrm{g}}$. For these observations, the only thing we can mention is the fact that the temperatures giving $J_{\mathrm{av}}{ }^{\text {max }}$ peaks are reproducibly higher those giving $<\tau_{\mathrm{R}}>$ peaks in two pairs of heating and cooling experiments, and that $C_{\mathrm{av}}(\tau)$ oscillating and damping characteristics have different but a related physical origin (see Sub-second frequencies in power spectra in Discussion).

We consider that the peak at $29.6{ }^{\circ} \mathrm{C}$ in Figure $6 \mathrm{E}$ and the bump at $64.6{ }^{\circ} \mathrm{C}$ in Figure $6 \mathrm{~F}$ are not essential because no such peak and bump were reproducibly observed in another pair of heating and cooling experiments in Figures 6I and 6J (see Discontinuous change in temperaturedependent relaxation time above $T_{g}$ in Discussion). Aside from these irregularities, we commonly observed $<\tau_{\mathrm{R}}>$ peaks at specific temperatures $T_{\mathrm{c}}=37.8\left(46.4{ }^{\circ} \mathrm{C}\right)$ and $T_{\mathrm{c}}{ }^{\prime}=41.6\left(32.8{ }^{\circ} \mathrm{C}\right)$, respectively above $T_{\mathrm{g}}$. These are essential observations in temperature dependent $<\tau_{\mathrm{R}}>$. Increasing 
$<\tau_{\mathrm{R}}>$ with decreasing temperature below $T_{\mathrm{g}}=36.5{ }^{\circ} \mathrm{C}$ is reasonable considering increased viscosity at lower temperatures and $\mathrm{Cy} 3$ viscosity-sensitive nature.
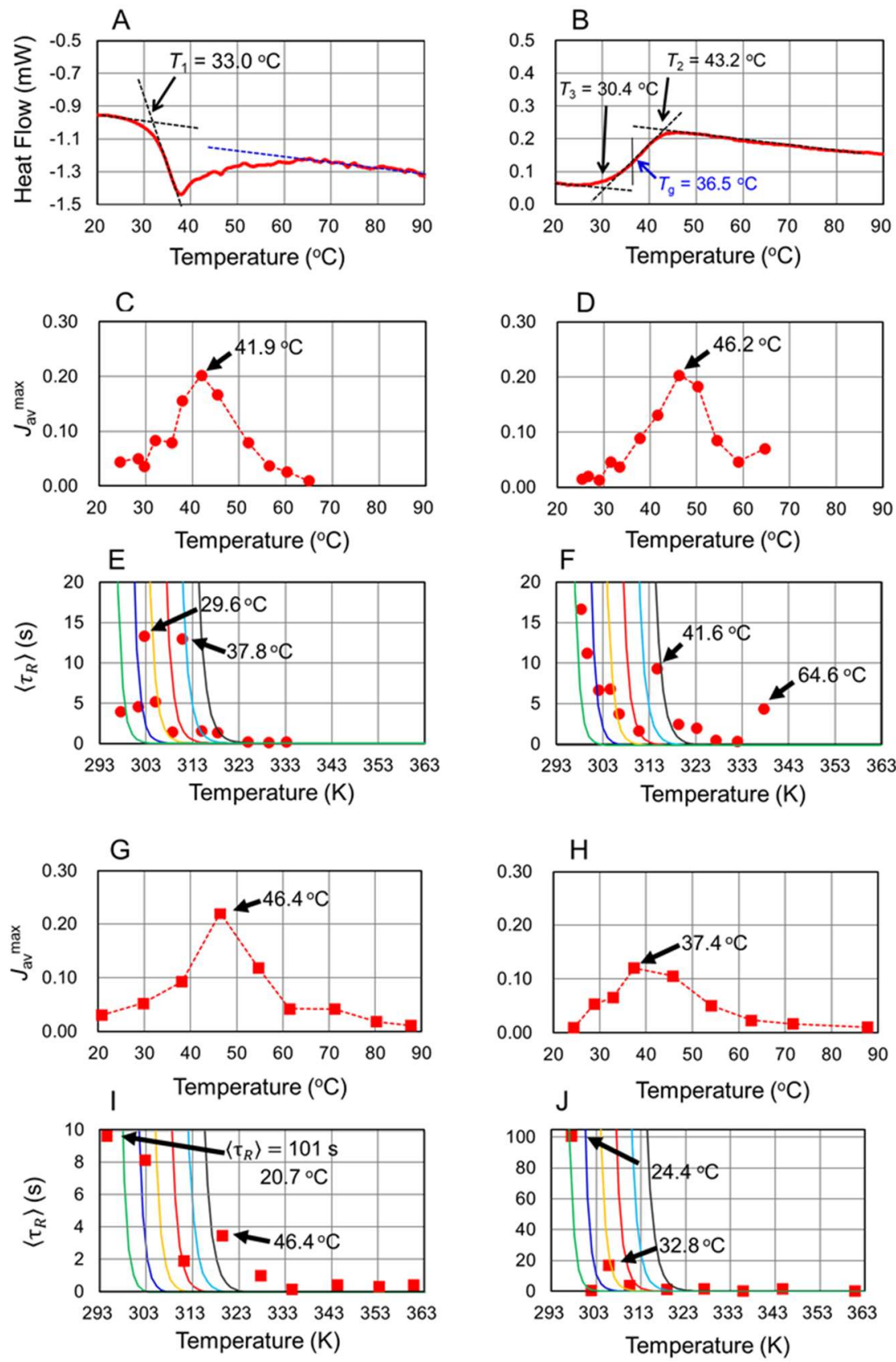

Figure 6. Temperature dependent DSC measurements; maximum average power spectra, $J_{\mathrm{av}}{ }^{\max }$; and temperature dependent average relaxation time, $<\tau_{\mathbf{R}}>$ superposed with VFT lines: DSC traces for (A) heating and (B) cooling $(5.0 \mathrm{~K} / \mathrm{min}) ; J_{\mathrm{av}}{ }^{\max }(\mathrm{v})$ for $(\mathbf{C})$ heating $(0.2 \mathrm{~K} / \mathrm{min})$ and $(\mathbf{D})$ cooling $(0.2-0.3 \mathrm{~K} / \mathrm{min})$; $<\tau_{\mathrm{R}}>$ for (E) heating and (F) cooling $(0.2-0.3 \mathrm{~K} / \mathrm{min})$. VFT lines $(B=460,510,560,610,660$, and $710 \mathrm{~K}$ in green, blue, orange, light blue, and gray, respectively, computed from eq 4 using each $B$ value) are superposed on the $<\tau_{\mathrm{R}}>$ plot. $J_{a v}{ }^{\max }(v)$ is Fourier transform of $C_{\mathrm{av}}(\tau)$, average twenty $C(\tau)$ traces, at each temperature. From another pair of heating and cooling experiments, we obtained $(\mathbf{G})$ and $(\mathbf{H})$. They are counterparts of $(\mathbf{C})$ and $(\mathbf{D})$, respectively; similarly, (I) and (J) are those of $(\mathbf{E})$ and $(\mathbf{F})$, respectively. (A) and (B) show temperature zone switching from glass to melt and vice versa between $T_{1}=33.0 \approx T_{2}=30.4$ and $T_{3}=43.2^{\circ} \mathrm{C}$ including $T_{\mathrm{g}}=36.5^{\circ} \mathrm{C}$. VFT lines for $B=610,660$, and $710 \mathrm{~K}$ were experimentally identified. ${ }^{29}$ For heating $24.5,28.3,29.6,32.0,35.6,37.8,41.9$, $45.4,51.9,56.4,60.2$, and $64.9^{\circ} \mathrm{C}$ at $0.2 \mathrm{~K} / \mathrm{min}$; for cooling $64.6,58.9,54.3,50.2,46.2,41.6,37.7,33.4,31.5$, $29.0,26.6$, and $25.3{ }^{\circ} \mathrm{C}$ at $0.2-0.3 \mathrm{~K} / \mathrm{min}$. 
To find another viewpoint of $<\tau_{\mathrm{R}}>$ peaks at 37.8 and $41.6^{\circ} \mathrm{C}$ in Figures $6 \mathrm{E}$ and $6 \mathrm{~F}$, respectively, we computed VFT lines using another VFT equation

$$
\log \left(\omega_{\infty} / \omega_{\max }\right)=B /\left(T-T_{o}\right)
$$

where $\omega_{\infty}$ is the limiting angular velocity at infinite temperature, using $B=660 \pm 50 \mathrm{~K}^{29}$ in addition to other three $B$ values we selected, and then converted $\omega_{\max }=2 \pi v$ into $\left\langle\tau_{\mathrm{R}}\right\rangle$ from $1 / v=\left\langle\tau_{\mathrm{R}}\right\rangle$. The $\omega_{\max }$ into $<\tau_{\mathrm{R}}>$ conversion is reasonable considering that $\omega_{\max }$ means resonance maximum in mechanical and dielectric loss spectroscopies. The computed VFT lines are superposed on Figures $6 \mathrm{E}$ and $6 \mathrm{~F}$. These figures suggest that something important occurs at 37.8 and $41.6{ }^{\circ} \mathrm{C}$. For example, in the cooling experiment (Figure $6 \mathrm{~F}$ ) $<\tau_{\mathrm{R}}>$ initially followed a VFT line (black, $B=$ $710 \mathrm{~K}$ ) and then suddenly altered its course within a zone enclosed by VFT lines (green, blue, yellow, and red, $B=460,510,560$, and $610 \mathrm{~K}$, respectively). This is just another viewpoint of the second major finding characterized by discontinuous $<\tau_{\mathrm{R}}>$ transition from one VFT line to others near $T_{\mathrm{g}}$. In this sense, the $<\tau_{\mathrm{R}}>$ peaks are VFT line transition edges. We further argue the peaks or the edges in terms of critical slowing down in Discontinuous change in temperature-dependent relaxation times above $T_{g}$ in Discussion.

Figures 6A-6F compare DSC and SMS observations considering two experimental conditions differences: surface and interface effects on $T_{\mathrm{g}}$ for polymer thin films, ${ }^{28}$ which generated different $T_{\mathrm{g}}$ from bulk polymer samples, and heating and cooling rate differences $(5.0$ and $0.2-0.3 \mathrm{~K} / \mathrm{min}$ for DSC and SMS, respectively). We used 4.0-8.0 mg PVAC powder as bulk PVAC for DSC, and a 300-400 nm thick PVAC film for SMS. Despite these experimental condition differences, a close relationship is reasonable between thermal properties evaluated by DSC and active collective motions occurrences for PVAC observed by SMS in the sense that $J_{\text {av }}(v)$ amplitude reached the top between $T_{\mathrm{g}}$ and $T_{\mathrm{g}}+10 \mathrm{~K}$, at which glass transition begin to start in cooling experiment. This means that $T_{\mathrm{g}}$ is likely close to glass transition temperatures probed by singlemolecule Cy3 at interfaces $T_{\mathrm{g}}{ }^{\text {int }}$ (for more detail see Single Cy3 molecules sensing PVAC specific temperatures at interfaces in Supplementary Text in $S I$ ).

Summarizing this section, temperature dependent $J_{\text {av }}{ }^{\max }$ (Figures $6 \mathrm{C}$ and $6 \mathrm{D}$ ) disclosed a temperature window between 30 and $60^{\circ} \mathrm{C}$, including $T_{\mathrm{g}}=36.5^{\circ} \mathrm{C}(309.5 \mathrm{~K})$ evaluated by DSC cooling (Figure 6B). Temperature dependent $\left\langle\tau_{R}\right\rangle$ (Figures $6 \mathrm{E}$ and $6 \mathrm{~F}$ ) pinpointed specific temperatures within the $30-60{ }^{\circ} \mathrm{C}$ window, showing the second major finding. These temperatures are considerably less than $T_{\text {cross }} \approx 1.2 T_{\mathrm{g}}=371.4 \mathrm{~K}\left(98.4{ }^{\circ} \mathrm{C}\right)$. Thus, the temperature window characterized by highly fluctuating collective PVAC motions embodied by the $C(\tau)$ cosine waveform appears outside MCT but involves cooperative $\alpha$ process active zone

\section{DISCUSSION}

Here we develop arguments to help understand the two major findings outlined in Introduction and presented in Results. The first major finding was cosine waveform $C(\tau)$ in Figures 3C, 3D, $\mathrm{S} 4 \mathrm{~B}$, and S4C. This waveform was derived from GLE under the approximation leading to the understanding that PVAC environment coherently and persistently influences viscosity sensitive single Cy3 molecule fluorescence. The second major finding was temperature dependent $\left\langle\tau_{\mathrm{R}}>\right.$ showing discontinuous change (Figures 5E and 5F), which is similar to the critical slowing down in the second-order phase transition.

Before moving on to the second major finding discussion, sub-second frequency origins in $J(v)$ (Figures 3B-3D, 3F, S4B-S4D, and S4F) was examined to understand the relationship between the sub-second frequencies in the first major finding and $\left\langle\tau_{\mathrm{R}}>\right.$ discontinuous change in the second major finding. Additionally, we present a scheme to explain $I_{\mathrm{f}}(t)$ fluctuation and $\mathrm{Cy} 3$ photophysics behind $I_{\mathrm{f}}(t)$ fluctuation (Figures S6A-S6D), and then give consideration to ascertain the validity of single $\mathrm{Cy} 3$ molecules sensing PVAC environment and glass transition at interfaces (Figures S2A, S2B, and Figures S3A-S3C) in Supplementary Text in SI. 


\section{Derivation of the $\mathbf{C}(\tau)$ cosine waveform from generalized Langevin equation}

The normalized autocorrelation function $C(\tau)$ is defined as

$$
C(\tau)=R(\tau) / R(0),
$$

where $R(\tau)$ is the autocorrelation function and $R(0)$ is a normalizing factor. Before evaluating $C(\tau)$ we computed $R(\tau)$ from $I_{\mathrm{f}}(t)$

$$
R(\tau)=\frac{1}{N} \sum_{i}^{N} I_{f}^{o}(i) I_{f}^{o}(i+\tau) \equiv\left\langle I_{f}^{o}(i) I_{f}^{o}(i+\tau)\right\rangle
$$

where $I_{\mathrm{f}}^{\mathrm{o}}(i)=I_{\mathrm{f}}(i)-\bar{I}_{\mathrm{f}} ; \bar{I}_{\mathrm{f}}$ is average $I_{\mathrm{f}}(t)$ over 512 frames, $N=256$ is the number of data points (half the number of the total frames) with step size or bin time $\Delta t=36 \mathrm{~ms}, i$ and $\tau$ are running suffix for each data point.

We summarize cosine autocorrelation function derivation ${ }^{30}$ from the GLE $^{26}$

$$
\frac{d A(t)}{d t}=i \omega_{o} A(t)-\int_{0}^{t} \varphi\left(t-t^{\prime}\right) A\left(t^{\prime}\right) d t^{\prime}+f(t),
$$

where $A(\mathrm{t})$ is a dynamical variable (generally a vector), $\omega_{\mathrm{o}}$ is a formal angular frequency (generally a matrix with zero diagonal elements), $f(\mathrm{t})$ is a randomly fluctuating external force, and $\varphi\left(t-t^{\prime}\right)$ is a memory function combining $A(\mathrm{t})$ at $t^{\prime}$ (past) and $t$ (present) given by

$$
\varphi\left(t-t^{\prime}\right)=\frac{\left\langle f(t) f\left(t^{\prime}\right)\right\rangle}{\langle f(0) f(0)\rangle},
$$

Equation 8 is an essential result from GLE formulation, ${ }^{26}$ signifying that the memory function is explicitly combined with normalized external force time correlation function. This equation is also a kind of fluctuation dispersion theorem. For the present derivation, $A(\mathrm{t})$ is a scaler variable equivalent to $I_{\mathrm{f}}(t)$; hence $\omega_{\mathrm{o}}=0$ because it is a $[1 \times 1]$ dimensional matrix with zero diagonal.

GLE is applicable to Brownian motion analyses, and its use here is quite reasonable considering that polymer dynamics are modeled as polymer segment Brownian motion. ${ }^{21}$ After several derivation steps from eq 7 (the skipped derivations are provided in $S I$ ),

$$
\frac{d \Phi(t)}{d t}=-\omega^{2} \int_{0}^{t} \varphi\left(t-t^{\prime}\right) \Phi\left(t^{\prime}\right) d t^{\prime},
$$

where $\omega^{2}$ is constant and $\Phi(\mathrm{t})$ is a normalized autocorrelation function equivalent to $C(\tau)$,

$$
\Phi(t)=\frac{\langle A(t) A(0)\rangle}{\langle A(0) A(0)\rangle},
$$

where $<A(\mathrm{t}) A(0)>$ is an autocorrelation function of $A(\mathrm{t})$. Substituting $\varphi\left(t-t^{\prime}\right)=1$ into eq 9 , that is, no memory function extinction approximation, ${ }^{30}$ gives

$$
\frac{d \Phi(t)}{d t}=-\omega^{2} \int_{0}^{t} \Phi\left(t^{\prime}\right) d t^{\prime}
$$

and hence

$$
\Phi(t)=\cos \omega t
$$

because $\Phi(0)=1$ from the normalized autocorrelation function definition. Thus, we have a cosine autocorrelation function with no memory function extinction approximation. This provides the rationale for cosine $C(\tau)$ waveforms (Figures $3 \mathrm{C}$ and $\mathrm{S} 4 \mathrm{C}$ ) and can be explained as follows.

No memory function extinction means $\left\langle f(t) f\left(t^{\prime}\right)\right\rangle \approx \varphi\left(t-t^{\prime}\right)=$ constant, that is, $f(t)$ shows how PVAC collective motion coherently and persistently affects single Cy3 molecules as Brownian particles. On the other hand, instantaneous $\varphi\left(t-t^{\prime}\right)$ disappearance approximation provides a single exponential decay $C(\tau)$. Damped oscillation (Figures $3 \mathrm{~F}$ and $\mathrm{S} 4 \mathrm{~F}$ ) as an intermediate 
waveform between cosine and single exponential decay was dominantly observed in the present work. Figures S7A and S7B compare the two approximations for GLE in SI.

\section{Sub-second frequencies in power spectra}

Frequencies lower than $1 \mathrm{~Hz}(0.109-0.542 \mathrm{~Hz}$, Figures 3B-3D and 3F; and $0.217-0.868 \mathrm{~Hz}$, Figures S4B-S4D and S4F) occurred for $J(v)>0.05$. In this section, we investigate which factors determined these sub-second frequencies. From the PVAC collective motion idea derived from $C(\tau)$ cosine waveforms, we expect PVAC viscoelasticity controlling the collective motion. Polymer including PVAC viscoelastic properties have widely been investigated by mechanical and dielectric spectroscopy ${ }^{29}$ A function to evaluate viscoelasticity is the shear modulus $G^{*}(\omega)=$ $G^{\prime}(\omega)+\mathrm{i} G^{\prime \prime}(\omega)$, which is evaluated by applying sinusoidal shear strain $\Gamma=\Gamma_{\mathrm{o}} \sin \omega t$ to polymers under the linear response condition.

A function $G^{\prime}(\omega)$, shear storage or elastic modulus, means applied shear strain energy storage in polymers and shows a blunt-edged ascending staircase shape in a $\log \omega$ vs $G^{\prime}(\omega)$ graph. The other function $G^{\prime \prime}(\omega)$, shear loss modulus, represents applied shear strain energy dissipation, or relaxation in polymers as heat, and takes a maximum loss peak at $\omega_{\max }$ in a $\log \omega$ vs $G^{\prime \prime}(\omega)$ presentation. A possibility that $G^{\prime}(\omega)$ is involved in the sub-second frequencies (Figures 3C and $\mathrm{S} 4 \mathrm{C})$ is ruled out due to the fact that $G^{\prime \prime}(\omega)$ denotes energy loss, or relaxation; relaxation is independent of oscillation in damped oscillations. For instance, the cosine waveform in Figures $3 \mathrm{C}$ oscillates in $0.217 \mathrm{~Hz}$ but its decay time is $15 \mathrm{~s}$.

We now consider the oscillation character in Figures 3B-3D, 3F, S4B-S4D, and S4F based on $G^{\prime}(\omega)$. The function $G^{\prime}(\omega)$ means much elastic energy storage in polymers having larger elasticity in higher frequencies in an opposite way that capacitors having higher dielectric permittivity $\varepsilon^{\prime}(\omega)$ (larger charge displacement in lower frequencies) store much electric energy. As noted in Type I$\mathrm{V}$ classification in Results, we found that the frequencies in $J(v)$ increased with temperature rise. This observation is consistent with the fact that polymers softening, or $G^{\prime}(\omega)$ reduction by heating, moves $G^{\prime}(\omega)$ curves to higher frequencies. In short, the frequencies in damped oscillations very likely reflect frequencies involved in $G^{\prime}(\omega)$.

In principle, $G^{\prime}(\omega)$ as well as $G^{\prime}(\omega)$ responds extremely wide frequencies as symbolized by $G^{\prime}(\omega)$ or $G^{\prime}(\omega)$ versus $\log \omega(-5$ to +10 at the most $)$ presentation. We employed $36 \mathrm{~ms}$ bin time and a 256 -frame temporal window $(9.180 \mathrm{~s}$, its reciprocal equals $0.109 \mathrm{~Hz})$ for $\left\langle\tau_{R}>\right.$ evaluation, limiting the frequency range we observed. The SMS time window is extremely limited compared with that of mechanical and dielectric spectroscopy. However, the SMS time window allows us to observe unusual phenomena, such as observations in Figures 3A-3F and S4A-S4F, missed by mechanical and dielectric spectroscopy.

\section{Discontinuous change in temperature-dependent relaxation time above $\boldsymbol{T}_{\mathrm{g}}$}

We examine the significance of $<\tau_{\mathrm{R}}>$ discontinuous change, peaks or edges, at $37.8^{\circ} \mathrm{C}$ and $41.6^{\circ} \mathrm{C}$ in Figures $6 \mathrm{E}$ and $6 \mathrm{~F}$. These temperatures fall within the temperature window $\left(30-60^{\circ} \mathrm{C}\right)$ where $J_{\mathrm{av}}{ }^{\max }(v)$ was enhanced (Figures 6C and 6D). Higher $J_{\mathrm{av}}{ }^{\max }(v)$ amplitude tended to be associated with activated $C_{\text {av }}(\tau)$ cosine or oscillatory characteristics (Figures $5 \mathrm{~A}-5 \mathrm{~L}$ and $\mathrm{S} 5 \mathrm{~A}-\mathrm{S} 5 \mathrm{~L}$ ). We thus consider two temperatures 37.8 and $41.6{ }^{\circ} \mathrm{C}$ to be $T_{\mathrm{c}}$ and $T_{\mathrm{c}}$ ' from heating and cooling $<\tau_{\mathrm{R}}>$ measurements, respectively, from the following discussion.

Perfect cosine $C(\tau)$ without damping shows infinite $\tau_{\mathrm{R}}$. Similarly, $<\tau_{\mathrm{R}}>$ exhibited peaks at $T_{\mathrm{c}}=$ 37.8 and $T_{\mathrm{c}}{ }^{\prime}=41.6{ }^{\circ} \mathrm{C}$ within the $30-60{ }^{\circ} \mathrm{C}$ temperature window in which $C_{\text {av }}(\tau)$ cosine characteristics (collective PVAC motion) were noticeable. Thus, the two peaks are similar to order parameter discontinuity in the second-order phase transition or critical phenomena ${ }^{17}$ typified by critical opalescence showing large collective density fluctuations in $\mathrm{H}_{2} \mathrm{O}$ or $\mathrm{CO}_{2}$ showing opaque appearance. Considering $C(\tau)$ diversity before averaging (Figures 4A and 4B; Tables S2A and S2B), the fact that $\left\langle\tau_{\mathrm{R}}>\right.$ evaluated from $C_{\mathrm{av}}(\tau)$ is far from infinite $\tau_{\mathrm{R}}$ is understandable. Nevertheless, $<\tau_{\mathrm{R}}>$ showed discontinuous changes associated with PVAC collective motion. Thus, 
they look critical slowing down considering that they occurred along with the activated collective motion, which is a critical phenomenon associated with the second-order phase transition.

Critical slowing down provides divergent $\tau_{\mathrm{R}}$ near $T_{\mathrm{c}}{ }^{31}$

$$
\tau_{R} \propto \frac{1}{\left(T-T_{c}\right)^{\gamma}},
$$

where $\gamma$ is a critical-point exponent. A moving particle on a surface models critical slowing down as follows. ${ }^{32}$ Consider a particle on the Gibbs potential designated by pressure $p$ and $T$ having two minima. Below $T_{\mathrm{c}}$ changing $p$ or $T$ allows the particle to move from one higher minimum to the lower one, generating the first-order phase transition. At $T_{\mathrm{c}}$ the second-order phase transition occurs; two potential minima are merged into one and the one-minimum potential is flattened, which is formulated in Landau theory. Thus, the particle moves slowly and widely on the flattened potential surface. The slowly moving particle models critical slowing down.

Equation 13 is equivalent to that of temperature dependent isothermal magnetic susceptibility $\chi_{\mathrm{T}}$ in magnets and isothermal compressibility $\kappa_{\mathrm{T}}$ in fluids. The $\gamma$ value is unity from the meanfield theory and Landau theory, but experimentally $1.2-1.4 .{ }^{17},{ }^{32}$ We restrict serious $\gamma$ value evaluation from Figures $6 \mathrm{E}$ and $6 \mathrm{~F}$ in the present article; however, $\gamma=0.5$ was unrealistic but $\gamma$ $=1$ was possible, and discrimination between $\gamma=1$ and $\gamma=1.5$ was hard to do in the limited number of data points in the tentative evaluation from these figures. The present article mission does not involve rigorous $\gamma$ evaluation but SMS application to glass transition issue elucidation. Experimental challenges to estimate more rigorously $\gamma$ values are now underway. Thus, the second-order phase transition behavior was observed not only in cosine $C(\tau)$ but also in temperature dependent discontinuous $<\tau_{\mathrm{R}}>$ changes.

We found two irregularities regarding $\left\langle\tau_{R}\right\rangle$ : an additional peak occurred below $30{ }^{\circ} \mathrm{C}$ for heating (Figure 6E) and the point indicated at $64.6^{\circ} \mathrm{C}$ for cooling (Figure $6 \mathrm{~F}$ ). The first irregularity is against the expectation that $<\tau_{\mathrm{R}}>$ increases with decreasing temperature below $T_{\mathrm{g}}$. One possible explanation for the decreased $<\tau_{\mathrm{R}}>$ is as follows. The two $<\tau_{\mathrm{R}}>$ data below $30^{\circ} \mathrm{C}$ (Figure $6 \mathrm{E}$ ) are due to the fact that single Cy3 molecules simply probed $<\tau_{R}>$ shorter than the $<\tau_{R}>$ expected from VFT lines. We remember that $\tau_{\mathrm{R}}$ from VFT lines comes from dielectric loss maxima $\omega_{\max }$ in a $\log$ $\omega$ vs $G$ " $(\omega)$ spectrum. This means that $<\tau_{R}>$ takes a value close to a VFT line or not from extremely wide $\tau_{R}$ possibilities within a limit of time window $(9.18 \mathrm{~s})$ for $<\tau_{R}>$ evaluation. The second irregularity is characterized by $\left\langle\tau_{R}\right\rangle$ larger than expectation at $64.6{ }^{\circ} \mathrm{C}$ and reflected by decreased $C(\tau)$ in Type $\mathrm{V}$ (Figures $4 \mathrm{~A}$ and $4 \mathrm{~B}$ ). The second irregularity looks accidental one simply due to an uncontrollable heterogeneous PVAC structures. Another pair of heating and cooling experiments (Figures 6I and 6J) supports the above understanding of the irregularities, where $\left\langle\tau_{R}>\right.$ increased with decreasing temperature below $40{ }^{\circ} \mathrm{C}$ and $\left\langle\tau_{R}\right\rangle$ decreased with increasing temperature above $60^{\circ} \mathrm{C}$.

The temperature dependent $\left\langle\tau_{\mathrm{R}}>\right.$ disclosed discontinuous $\left\langle\tau_{\mathrm{R}}>\right.$ changes near $T_{\mathrm{g}}$ before full PVAC solidification below $30{ }^{\circ} \mathrm{C}$, which is close to $T_{1}$ and $T_{3}$ in the DSC heating and cooling measurements (Figures 6A and 6B). Discontinuous change in $\left\langle\tau_{R}\right\rangle$ was reproduced in several pairs of heating and cooling SMS experiments; however, the temperatures showing the peak $\left\langle\tau_{R}>\right.$ were different from heating to cooling and experiment to experiment. The varied $\left\langle\tau_{R}>\right.$ peak occurrences can be rationalized as follows. In the present SMS experiments, we employed surface immobilized sub-nanometer size $\mathrm{Cy} 3$ probes, allowing spatially resolved measurement in the nanometer region. From the observations in Figures 4A (Table S1A) and 4B (Table S1B) showing that $C(\tau)$ in Type III, which indicates two major findings, did not exclusively occur within 30-60 window especially $T_{\mathrm{g}}+10 \mathrm{~K}$, we assume that not all the probed sites in PVAC present secondorder phase transition behavior and the sites that present second-order phase transition behavior do not simultaneously present second-order phase transition behavior. Note that $\left\langle\tau_{R}\right\rangle$ was evaluated from $C_{\text {av }}(\tau)$ averaged over every $C(\tau)$ including Type I to Type $\mathrm{V}$ at each temperature, likely generating varied $<\tau_{\mathrm{R}}>$ peaks. 
An early model, ${ }^{33}$ in which super cooled liquids include dispersed micro-crystals, inspires the idea of specific sites showing second-order phase transition behavior in PVAC. This model predicts the widely known empirical relationship $T_{\mathrm{g}} / T_{\mathrm{m}} \simeq 2 / 3$, making this model reliable. For this reason, our assumption considering the sites specific to second-order phase transition behavior in PVAC seems to be far from groundless.

\section{CONCLUSIONS}

We identified two sets evidence for second-order phase transition behavior in PVAC glass transition: highly active PVAC collective motions from $C(\tau)$ cosine waveforms above $T_{\mathrm{g}}$ and discontinuous transition in temperature dependent $\left\langle\tau_{R}\right\rangle$. These occurred within a specific temperature window between 30 and $60{ }^{\circ} \mathrm{C}$, or more exclusively for $\left\langle\tau_{\mathrm{R}}>\right.$ between $T_{3}=30.4{ }^{\circ} \mathrm{C}$ and $T_{2}=43.2{ }^{\circ} \mathrm{C}$ including $T_{\mathrm{g}}=36.5^{\circ} \mathrm{C}$, and below $T_{\text {cross }} \approx 1.2 T_{\mathrm{g}}=98.4{ }^{\circ} \mathrm{C}$.

Second-order phase transition behavior in glass transition has been suggested in temperature dependent $S, C_{\mathrm{p}}$, and $\eta$ as noted in Introduction. These parameters temperature dependence is solid experimental facts. However, such knowledge has lacked straightforward evidence for critical phenomena in glass transition mainly due to experimental limitations. The present achievements have disclosed critical phenomena in glass transition based on molecular-level experimental observations and made a breakthrough to settle a major unresolved problem in physics and chemistry.

The major findings arose from SMS, which is an unconventional method for glass transition investigation in contrast to the more widely used DSC, dielectric and mechanical spectroscopy, and so on. However, SMS in the present our status has one inability and one technical limitation in return for the uniqueness demonstrated in the present article. The inability is that no direct $\xi_{\alpha}$ evaluation but no critical opalescence observation from PVAC, suggesting $\xi_{\alpha}<100-200 \mathrm{~nm}$. The limitation is photobleaching in $\mathrm{Cy} 3$, or any fluorescent dyes. This issue prevents repeated video imaging, which limits the number of $<\tau_{\mathrm{R}}>$ data points.

\section{ASSOCIATED CONTENT \\ Corresponding Author \\ *E-mail: mi195526@josai.ac.jp \\ ORCID}

Mitsuru Ishikawa: 0000-0001-9438-2270

Takayuki Uwada: 0000-0002-4272-7964

Funding: This work was supported by graduate and undergraduate student research budgets in Department of Chemistry, Josai University; no other funding was involved such as Kaken-hi (MEXT) in the present work. Author contributions: M. I. planned this work, supervised three students, T. T., Y. H., and M.A. and wrote the manuscript. T. T. joined this work for three years as an undergraduate and a graduate student, contributing to experiment and data analysis. Y. H. and M. A. also joined this work for one year each as undergraduate students and contributed to part of experiment and data analysis. T. U. also supervised T. T. to complete his master's thesis involving this work. Competing interests: All the authors have no competing interests. Data and material availability: All data necessary for evaluating the conclusions in the present article are included in the manuscript and SI.

\section{ACKNOWLEDGMENTS}

M. I. thanks Prof. Vasudevan Pillai Biju, Research Institute for Electronic Science, Hokkaido University, for his kind advice and support for sample preparation, and also for permitting the use of Figures S6A-S6C, the original of which was prepared by himself. Also, Prof. Yoshihiro Tokudome, Department of Pharmaceutical Sciences, Josai University, is appreciated by M. I. for his technical advice for DSC equipment operation. 


\section{REFERENCES AND NOTES}

1. Single molecule spectroscopy, Nobel conference lectures, Eds. R. Rigler, M. Oritt, T. Basché, Springer 2001.

2. L. Kisley, C. F. Landes, Molecular approaches to chromatography using single molecule spectroscopy. Anal. Chem. 87, 83-98 (2015).

3. S. Ray, J. R. Widom, N. G. Walter, Life under the microscope: single-molecule fluorescence highlights the RNA world. Chem. Rev. 118, 4120-4155 (2018).

4. J. C. Scaiano, A. E. Lanterna, Is single-molecule fluorescence spectroscopy ready to join the organic chemistry toolkit? A test case involving click chemistry. J. Org. Chem. 82, 5011-5019 (2017).

5. T. Tachikawa, S. Yamashita, T. Majima, Evidence for crystal-face-dependent $\mathrm{TiO}_{2}$ photocatalysis from single-molecule imaging and kinetic analysis. J. Am. Chem. Soc. 133, 7197-7204 (2011).

6. M. Ishikawa, J. Y. Ye, Y. Maruyama, H. Nakatsuka, Triphenylmethane dyes revealing heterogeneity of their nanoenvironment: femtosecond, picosecond, and single-molecule studies. J. Phys. Chem. A 103, 4319-4331 (1999).

7. V. P. Biju, J. Y. Ye, M. Ishikawa, Spatial heterogeneity in a polymer thin films probed by single molecules. J. Phys. Chem. B 107, 10729-10735 (2003).

8. N. Adhikari, N. A. Capurso, D. Bingermann, Heterogeneous dynamics and dynamic heterogeneities at the glass transition probed with single molecule spectroscopy. J. Chem. Phys. 127, 114508 (2007).

9. T. Oba, M. Vach, Relaxation in thin films mapped across the film thickness by astigmatic single-molecule imaging. ACS Macro Lett. 1, 784-788 (2012).

10. M. F. Serag, M. Abadi, S. Habuchi, Single-molecule diffusion and conformational dynamics by spatial integration of temporal fluctuations. Nat. Commun. 5, 5123 (2014).

11. P. G. Debenedetti, F. H. Stillinger, Supercooled liquids and the glass transition. Nature 410, 259-267 (2001).

12. P. W. Anderson, Through the glass lightly. Science 267, 1615-1616 (1995).

13. McKenna, G. B.; Simon, S. L. $50^{\text {th }}$ Anniversary perspective: challenges in the dynamics and kinetics of glass-forming polymers. Macromolecules 50, 6333-6361 (2017).

14. J. Y. Ye, T. Hattori, H. Inouye, H. Ueta, H. Nakatsuka, Y. Maruyama, M. Ishikawa, Glass transition of associated solvents studied by fluorescence measurement of doped chromophores. Phys. Rev. B 53, 8349-8353 (1996).

15. J. Y. Ye, T. Hattori, H. Nakatsuka, Y. Maruyama, M. Ishikawa, Microscopic dynamics of the glass transition investigated by time-resolved fluorescence measurements of doped chromophores. Phys. Rev. B 56, 5286-5295 (1997).

16. E. Donth, "1. Introduction" and "2.1. The classical picture. No serious problem" in The Glass Transition, Relaxation Dynamics in Liquid and Disordered Materials, (Springer 2001), pp 1-66.

17. H. E. Stanley, "What are the critical phenomena? A survey of some basic results" in Introduction to phase transitions and critical phenomena, (Oxford 1971), pp. 1-21.

18. C. A. Angell, K. L. Ngai, G. B. McKenna, P. F. McMillan, S. W. Martin, Relaxation in glassforming liquids and amorphous solids. J. Appl. Phys. 88, 3113-3157 (2000).

19. K. Miyazaki, M. Ozawa, A. Ikeda, Recent development of theory of the glass transition. Netsu Sokutei 42, 135-141(2015) (in Japanese).

20. H. Sillescu, Heterogeneity at the glass transition: a review. J. Non-Cryst. Solids $\mathbf{2 4 3}, 81-$ 108 (1999).

21. Segment is a notion intrinsic to polymer physics and is not equivalent to monomer but means a moving body composed of one to ten monomers. Polymer dynamics based on statistical mechanics regards a polymer chain as an ensemble of segments for mathematical 
convenience, each of which can be treated statistically independent particles. Thus, we can treat polymer dynamics as micro Brownian motion.

22. M. D. Ediger, Spatially heterogeneous dynamics in supercooled liquids. Annu. Rev. Phys. Chem. 51, 99-128 (2000).

23. U. Tracht, M. Wilhelm, A. Heuer, H. Feng, K. Schmidt-Rohr, H. W. Spiess, Length scale of dynamic heterogeneities at the glass transition determined by multidimensional nuclear magnetic resonance. Phys. Rev. Lett. 81, 2727-2730 (1998).

24. E. Hempel, G. Hempel, A. Hensel, C. Schick, E. Donth, Characteristic length of dynamic glass transition near $T_{\mathrm{g}}$ for a wide assortment of glass-forming substances. J. Phys. Chem. $B$ 104, 2460-2466 (2000).

25. K. Chibisov, G. V. Zakharova, H. Görner, Yu. A. Sogulyaev, I. L. Mushkalo, A. I. Tolmachev, Photorelaxation processes in covalently linked indocarbocyanine and thiacarbocyanine dyes. J. Phys. Chem. 99, 886-893 (1995).

26. H. Mori, Transport, collective motion, and Brownian motion. Prog. Theor. Phys. 33, 423455 (1965).

27. http://polymerdatabase.com/polymers/polyvinylacetate.html (accessed June 4, 2020). In this site we found that PVAC $T_{\mathrm{g}}$ ranges $297-315 \mathrm{~K}$ and $307 \mathrm{~K}$ is noted as a preferred value.

28. Y. Fuji-i, H. Morita, A. Takahara, T. Kanaya, "Mobility gradient of polystyrene in films supported on solid substrates" in Glass transition, dynamics and heterogeneity of polymer thin films, Ed. T. Kanaya (Springer, 2013), pp 1-28.

29. M. Beiner, J. Korus, H. Lockwenz, K. Schröter, E. Donth, Heat capacity spectroscopy compared to other linear response methods at dynamic glass transition in poly(vinyl acetate). Macromolecules 29, 5183-5189 (1996).

30. H. Shimizu, "Kanwa-gensyo no kiso (Fundamentals in relaxation phenomena)" in Kanwagensyo no kagaku (Relaxation phenomena in chemistry), Eds. K. Higashi, S. Nagakura (Iwanami, 1973), pp 5-73 (in Japanese).

31. S. K. Ma, "The Van Hove Theory" in "Modern theory of critical phenomena", (Westview, 1976), pp 442-449.

32. H. B. Callen, "Critical phenomena" in "Thermodynamics and introduction to thermostatistics" $2^{\text {nd }} \mathrm{Ed}$. (Wiley, 1985), pp 255-275.

33. W. Kauzmann, The nature of the glassy state and the behavior of liquids at low temperatures. Chem. Rev. 43, 219-256 (1948).

34. F. Köhn, J. Hofkens, R. Gronheid, M. Van der Auweraer, F. C. De Schryver, Parameters influencing the on- and off-times in the fluorescence intensity traces of single cyanine dye molecules. J. Phys. Chem. A 106, 4808-4814 (2002).

35. K. Jia, Y. Wan, A. Xia, S. Li, F. Gong, G. Yang, Characterization of photoinduced isomerization and intersystem crossing of the cyanine dye Cy3. J. Phys. Chem. A 111, 1593-1597 (2007).

36. N. J. Turro,"Photophysical radiationless transitions" in Modern Molecular Photochemistry (Addison-Wesley, ed. 2, 1978), pp 153-198.

37. S. Yu. Grebenkin, B. V. Bol'shakov, Cage effects upon light irradiation on azo compounds: cis $\rightarrow$ trans isomerization in polymethyl methacrylate. J. Photochem. Photobiol., A 122, 205-209 (1999).

38. H. Mortazavian, C. J. Fennell, F. D. Blum, Structure of the interfacial region in adsorbed poly(vinyl acetate) on silica. Macromolecules 49, 298-307 (2016). 